\title{
Dynamic Control Flow in Large-Scale Machine Learning
}

\author{
Yuan $\mathrm{Yu}^{*}$ \\ Microsoft \\ yuanbyu@microsoft.com \\ Eugene Brevdo \\ Google Brain \\ ebrevdo@google.com \\ Jeff Dean \\ Google Brain \\ jeff@google.com \\ Peter Hawkins \\ Google Brain \\ phawkins@google.com

$$
\begin{gathered}
\text { Rajat Monga } \\
\text { Google Brain }
\end{gathered}
$$ \\ rajatmonga@google.com
}

\author{
Martín Abadi \\ Google Brain \\ abadi@google.com \\ Mike Burrows \\ Google Brain \\ m3b@google.com \\ Sanjay Ghemawat
Google
sanjay@google.com \\ Michael Isard
Google Brain
misard@google.com \\ Derek Murray \\ Google Brain \\ mrry@google.com
}

\author{
Paul Barham \\ Google Brain \\ pbar@google.com \\ Andy Davis \\ Google Brain \\ andydavis@google.com \\ Tim Harley \\ DeepMind \\ tharley@google.com \\ Manjunath Kudlur
Cerebras Systems
manjunath@cerebras.net \\ Xiaoqiang Zheng \\ Google Brain \\ zhengxq@google.com
}

\begin{abstract}
Many recent machine learning models rely on fine-grained dynamic control flow for training and inference. In particular, models based on recurrent neural networks and on reinforcement learning depend on recurrence relations, data-dependent conditional execution, and other features that call for dynamic control flow. These applications benefit from the ability to make rapid control-flow decisions across a set of computing devices in a distributed system. For performance, scalability, and expressiveness, a machine learning system must support dynamic control flow in distributed and heterogeneous environments.

This paper presents a programming model for distributed machine learning that supports dynamic control flow. We describe the design of the programming model, and its implementation in TensorFlow, a distributed machine learning system. Our approach extends the use of dataflow graphs to represent machine learning models, offering several distinctive features. First, the branches of conditionals and bodies of loops can be partitioned across many machines to run on a set of heterogeneous devices, including CPUs, GPUs, and custom ASICs. Second, programs written in our model support automatic differentiation and distributed gradient computations, which are necessary for training machine learning models
\end{abstract}

${ }^{*}$ Work done primarily at Google Brain.

$(9) \Theta \Theta$

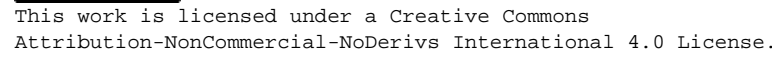

EuroSys '18, April 23-26, 2018, Porto, Portugal

(c) 2018 Copyright held by the owner/author(s)

ACM ISBN 978-1-4503-5584-1/18/04.

https://doi.org/10.1145/3190508.3190551 that use control flow. Third, our choice of non-strict semantics enables multiple loop iterations to execute in parallel across machines, and to overlap compute and $\mathrm{I} / \mathrm{O}$ operations.

We have done our work in the context of TensorFlow, and it has been used extensively in research and production. We evaluate it using several real-world applications, and demonstrate its performance and scalability.

\section{CCS CONCEPTS}

- Software and its engineering $\rightarrow$ Data flow languages; Distributed programming languages; Control structures; • Computing methodologies $\rightarrow$ Neural networks;

\section{ACM Reference Format:}

Yuan Yu, Martín Abadi, Paul Barham, Eugene Brevdo, Mike Burrows, Andy Davis, Jeff Dean, Sanjay Ghemawat, Tim Harley, Peter Hawkins, Michael Isard, Manjunath Kudlur, Rajat Monga, Derek Murray, and Xiaoqiang Zheng. 2018. Dynamic Control Flow in Large-Scale Machine Learning. In EuroSys '18: Thirteenth EuroSys Conference 2018, April 23-26, 2018, Porto, Portugal. ACM, New York, NY, USA, 15 pages. https://doi.org/10.1145/3190508.3190551

\section{INTRODUCTION}

Advances in machine learning and their many applications have brought into focus conflicting design objectives for the underlying systems. These systems should be scalable: they should use hardware resources efficiently, on platforms from individual phones to powerful datacenters that comprise CPUs, GPUs, and custom ASICs such as TPUs [21, 22]. At the same time, systems should be expressive and flexible to support both production and research.

As an example, consider the model architecture depicted in Figure 1. It is a simplified version of one employed at Google for language processing. This architecture includes two RNN (Recurrent Neural Network) layers, and a "mixture of experts" (MoE) that provides dynamic, learned connections between the RNN layers. 


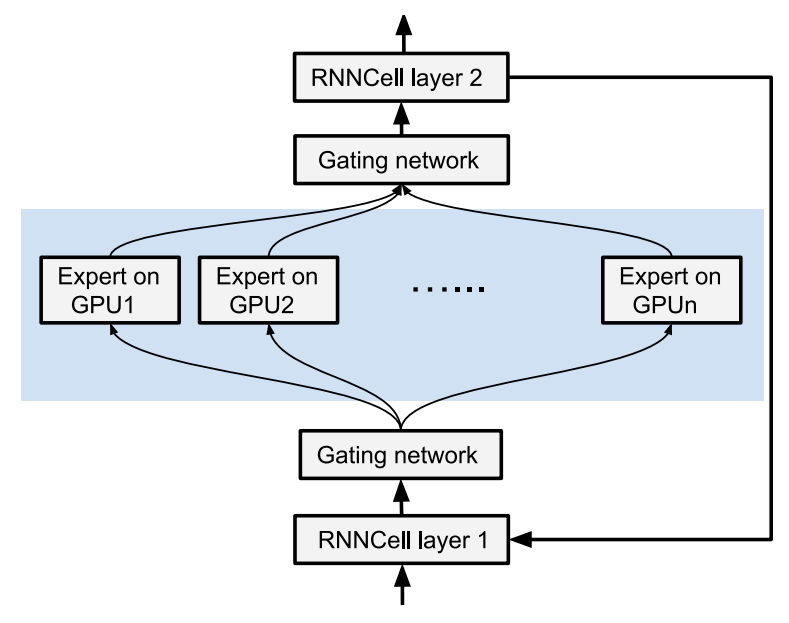

Figure 1: An example model architecture.

While simple feedforward neural networks are akin to straight-line code, this architecture makes essential use of dynamic control flow, with all the difficulties that it entails. An embodiment of the resulting system may run over a distributed infrastructure, with many components on different devices. Implementation choices can have a large impact on the performance of such a system and its memory requirements. For instance, RNNs give rise to challenging tradeoffs between memory footprint and computation time (e.g., [17]). Distributing computations over multiple GPUs and swapping tensors between GPU and host memory can alleviate these memory constraints, and adds another dimension to those trade-offs.

Both the building blocks of machine learning (e.g., individual cells in Figure 1) and the architectures built up using these blocks have been changing rapidly. This pace appears likely to continue. Therefore, rather than defining RNNs, MoEs, and other features as primitives of a programming model, it is attractive to be able to implement them in terms of general control-flow constructs such as conditionals and loops. Thus, we advocate that machine learning systems should provide general facilities for dynamic control flow, and we address the challenge of making them work efficiently in heterogenous distributed systems consisting of CPUs, GPUs, and TPUs.

Modern machine learning frameworks typically use dataflow graphs to represent computations, and a client process to drive those computations on a collection of accelerators. The frameworks offer two main approaches for control flow:

- The in-graph approach [1,2] encodes control-flow decisions as operations in the dataflow graph.

- The out-of-graph approach [10,12,20] implements controlflow decisions in the client process, using control-flow primitives of a host language (e.g., Python).

When designing TensorFlow [1] we favored the in-graph approach, because it has advantages at both compile and run time. With this approach, the machine learning system processes a single unified dataflow graph, and can perform whole-program optimization. In particular, it can determine exactly which intermediate values from the execution of a machine learning model are necessary to compute the gradients that are pervasive in machine learning algorithms, and it can decide to cache those values. In addition, this approach allows the entire computation to stay inside the system runtime during execution. This feature is important in heterogeneous environments where communication and synchronization with the client process can be costly.

If the in-graph dynamic control-flow features of TensorFlow are not used, a program must rely on the control-flow features of the host language, or resort to static unrolling. In-graph loops allow us to obtain more parallelism than out-of-graph loops. There can be a factor of 5 between the number of iterations per second in a microbenchmark that compares in-graph and out-of-graph loops distributed across 8 GPUs on one machine (Section 6.1). We explore and exploit these advantages in our work.

In our work, high-level control-flow constructs are compiled to dataflow graphs that include a few simple, powerful primitives similar to those of dynamic dataflow machines $[4,5]$. These graphs can then be arbitrarily partitioned and executed on a set of heterogeneous devices, using a lightweight coordination mechanism.

Since automatic differentiation is an important technique for training machine learning models, we also show how to support it efficiently in the presence of dynamic control flow. Given a graph, we demonstrate how to add a subgraph that computes gradients, and develop optimizations and memory-management techniques for this computation. If the original graph includes control-flow constructs, the new subgraph will as well. These subgraphs can also be partitioned and executed on a set of heterogeneous devices.

Our implementation allows parallelism and asynchrony, providing a non-strict semantics: operations in a conditional branch or a loop iteration can run as soon as their inputs are available. This property enables us to overlap the execution of control-flow logic on CPU, compute kernels on GPU, and memory-copy operations between CPU and GPU.

While it is difficult to measure ease-of-use or flexibility, we have evidence that our design and implementation are suitable for a variety of real-world needs. Our code is part of the core TensorFlow distribution, and is used widely in production and research. We analyzed more than 11.7 million unique graphs for machine learning jobs at Google over the past year, and found that approximately $65 \%$ contain some kind of conditional computation, and approximately $5 \%$ contain one or more loops. Teams that conduct research on model structures for machine learning, and several open-source projects (e.g., SyntaxNet [41], TensorFlow Fold [43], and Sonnet [39]) rely on our work. Accordingly, our evaluation of performance and scalability reflects results for real applications in the context of a real system.

The original TensorFlow paper [1] briefly sketched our approach to dynamic control flow, but did not provide a detailed design or evaluation. The present work goes much further in both respects. To the best of our knowledge, there is no previous dataflow system that supports both distributed control flow and automatic differentiation.

In sum, the main contributions of our work are:

- A design for how to incorporate in-graph dynamic control flow in machine learning, including, in particular, automatic differentiation. 
- A corresponding implementation that allows parallel and distributed execution across CPUs, GPUs, and custom machine learning accelerators.

- An evaluation that characterizes the performance and scalability of our techniques, and analyzes the impact of various choices.

- Extensive experience with users, which gives evidence of the expressiveness and flexibility of the system.

Section 2 discusses programming constructs for control flow and their applications in machine learning. Section 3 provides an architectural overview, highlighting challenges related to dynamic control flow. Section 4 describes our design and implementation. Section 5 considers two critical aspects of the system: automatic differentiation and memory management. Section 6 evaluates the performance and scalability of the system. Section 7 discusses related work.

\section{PROGRAMMING WITH CONTROL FLOW}

In this section, we briefly review TensorFlow's programming model and describe its support for dynamic control flow. We also discuss how dynamic control flow is being used in machine learning.

\subsection{Programming Interface}

TensorFlow employs a two-level programming model: programmers construct a dataflow graph using a high-level programming language; and the TensorFlow runtime takes a complete dataflow graph, optimizes it, and executes it on a set of devices. TensorFlow provides language bindings for many programming languages including Python, Java, and Go. In this paper, we use Python for illustration.

To simplify the task of constructing machine learning models, TensorFlow's API exposes a large collection of pre-defined operators. The operators are embedded as functions in the host programming languages. They support a wide range of mathematical operations on tensors, which are dense multi-dimensional arrays of basic data types such as float, integer, and string. The operators also include control-flow constructs, based on the work described in this paper. The most basic ones are cond and while_loop, for expressing conditional and iterative computation, respectively:

- $\operatorname{cond}(p r e d$, true_fn, false_fn) represents a conditional computation, where pred is a boolean tensor, and true_fn and false_fn are functions that construct the subgraphs for the respective branches. Both true_fn and false_fn return a tuple of tensors, with matching data types for each component; the result of cond is a tuple of tensors, representing the result of the branch that executes.

- while_loop(pred, body, inits) represents an iterative computation, where pred and body are functions that construct the subgraphs for the loop termination condition and the loop body; inits is a tuple of tensors that specifies the initial values of the loop variables. Both pred and body take a tuple of loop variables as arguments; pred returns a boolean tensor, and body returns a tuple of updated loop variables.

Other control-flow constructs include higher-order functions such as map_fn, foldl, foldr, and scan. However, the number of primitives remains small: the higher-order functions are actually defined

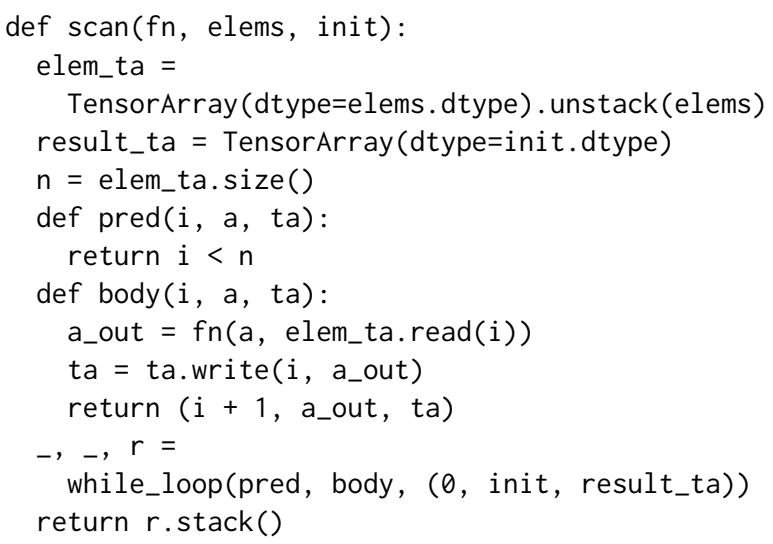

Figure 2: The scan operator can be defined using while_loop and TensorArrays.

in terms of while_loop. We support automatic differentiation for all these operators.

TensorFlow provides a few common data structures, including mutable variables and queues, and-as in conventional programsdata structures are an important tool for organizing a dynamic computation. TensorFlow initially lacked support for arrays of tensors with random read/write accesses, and we therefore augmented it with a new type of TensorArray objects. Unlike other data structures, TensorArrays can be used to store values consumed and produced by loops in a differentiable way, and they are a useful complement to loops. The basic operations on a TensorArray are ta. $\operatorname{read}(i x)$, which reads the array element at index $i x$, and ta. write (ix, $t$ ), which writes the value $t$ at index ix. A tensor may be converted to or from a TensorArray using the ta. unstack() and ta.stack() methods, respectively. We also support automatic differentiation for these operations (Section 5.2). TensorArrays are now used extensively in advanced machine learning models.

As an illustration of the expressiveness of our primitives, Figure 2 shows how we can define a higher-order scan, or generalized prefix-sum function, in terms of TensorArray objects and a while_loop(). Given a tensor elems with dimensions $[\mathrm{n}, \ldots .$.$] ,$ and an initial value init, scan computes a new tensor that contains the values of the expressions: $f n$ (init, elems $[0, \ldots]$ ), $f n(f n$ (init, elems $[0, \ldots]$ ), elems $[1, \ldots]$ ), ... It exemplifies a common computation pattern in machine learning: a tensor elems is first unstacked into a TensorArray of subtensors, elem_ta; a function $\mathrm{fn}$ is then repeatedly applied to those subtensors; and finally the results are packed back into a single tensor.

\subsection{Control Flow in Machine Learning}

Most traditional neural networks (including multi-layer perceptrons and convolutional neural networks) were static, in the sense that they were composed of a fixed number of layers, each with fixed operators. For learning over sequences (in particular, with RNNs), fixed-length iterations would typically be unrolled statically. In cases where some dynamic behavior was desired, the typical solution was to use client-side control-flow decisions. 
However, for the past few years, we have seen growing demand for dynamic control flow, especially in applications of recurrent and tree neural networks [3, 16, 24] and of reinforcement learning [26]. This section gives a few examples.

Beyond its programming-model implications, this trend raises the bar for implementations. For example, dynamic RNN models may operate over sequences of thousands of inputs. Since memory usage often grows linearly with sequence length, the amount of memory available on an accelerator is often the key factor that limits sequence length. Thus, this trend implies that memory optimizations are increasingly important. We address these challenges in later sections.

$R N N s$. The basic computation pattern of an RNN is to apply a cell function to every element of a sequence. A cell function takes as input a sequence element and the current "state" value; it returns an output sequence element and the updated state value, which is used when processing the next element. RNNs are thus well-suited for processing sequential data; some of their variants can also be applied to trees and other unbounded data structures.

RNNs are widely used in machine learning. One important application of RNNs is Neural Machine Translation (NMT) [40, 45], for which a model is composed of an encoder and a decoder. Both the encoder and decoder can conveniently be expressed as while-loops on variable-length sentences. We implemented the dynamic_rnn operator in TensorFlow using while-loops and TensorArray objects.

An RNN cell can be very compute-intensive, so we often need to distribute its execution across multiple devices. Some recent advances in RNN models have made the cell function itself perform a dynamic computation. For example, Adaptive Computation Time [16] uses a nested while-loop to learn how many computational steps to take at each time step of the outer loop of the RNNs. This application exercises our support for distributed execution of nested while-loops, and for their automatic differentiation.

Another example is the inclusion of an MoE layer inside the RNN cell [38]. Such a layer typically comprises a set of experts-simple neural networks that are specialized for a particular prediction task-and a gating function that chooses which experts to consult for a particular example. The experts and the gating function are subnetworks with trainable parameters. Since there can be a large number of experts (e.g., a few thousand in some NMT models) each typically with 1 million parameters, the experts are typically distributed across many machines.

Reinforcement learning. Reinforcement learning is a form of machine learning where an agent interacts with its environment by performing a sequence of actions according to some learned policy and receives rewards either at each interaction or after a sequence of interactions. The agent's goal is to choose actions that maximize the sum of these rewards.

Unlike in standard supervised learning, the agent's actions and the resulting rewards need not be deterministic or differentiable, so traditional backpropagation is insufficient and additional training techniques are required. These techniques generally benefit from dynamic control flow.

For example, some of our users write in-graph while-loops in which the agent interacts with its environment, repeatedly, and at each step the agent's actions are chosen by sampling according to learned probabilities. The expectation on the total rewards is a differentiable function of those probabilities, so can be optimized by gradient descent. Other users employ in-graph conditionals to dynamically read or write agent experiences to an in-graph database in parallel to the generation of the agent's actions. Some users also employ in-graph conditionals to create agents that choose whether to act randomly (explore) or act according to a learned policy (exploit).

Other usage. In addition to these examples of applications that emphasize machine learning architectures, we have seen several more mundane but useful applications of dynamic control flow. For instance, some of our users have relied on in-graph while-loops for programming training loops. (Generally, training loops are defined out-of-graph, in the host high-level programming language.) In this use case, a single coordinator process controls many workers that may not even be located in the same datacenter; in-graph control flow allows workers to make progress on training independently, without synchronizing with the coordinator between steps. Others have relied on in-graph conditionals for doing updates selectively, for example updating model parameters only when updates are sufficiently large, or updating only some model parameters in certain training steps.

\section{SYSTEM ARCHITECTURE}

In this section we review TensorFlow as a representative system architecture for a modern machine learning system [1], and discuss how dynamic control flow fits into it.

The core TensorFlow runtime is implemented in $\mathrm{C}++$ for portability and performance. The runtime exports a client API to front-ends for various languages (such as Python, Go, Java, and C++), whose role is to provide a high-level interface for building a dataflow graph, and manage the execution of that graph across a set of workers. In Section 4, we describe how the control-flow constructs in the client program are compiled into a dataflow graph representation for optimization and execution.

The TensorFlow runtime is responsible for the execution of the dataflow graph. It includes optimizations such as common subexpression elimination and constant propagation. To enable distributed execution on heterogeneous systems, a central coordinator automatically maps the nodes in the graph to the given set of devices, then partitions the graph into a set of subgraphs, one per device. When this partitioning would cut an edge between two devices, it automatically replaces the edge with a pair of communication operations, Send $(t, k)$ and $\operatorname{Recv}(k)$, which share a rendezvous key. After partitioning, each subgraph is shipped to the corresponding device, then executed by a device-local executor. The local executors communicate directly among themselves using Send and Recv operations, with no involvement from the central coordinator. When a tensor needs to be transported across devices, Send $(t, k)$ publishes the tensor $t$ under the rendezvous key $k$ on the sender's device, and $\operatorname{Recv}(k)$ pulls the tensor under key $k$ from the sender's device, blocking until it has been produced if necessary.

Dynamic control flow introduces additional challenges in the above design. Without control flow, each operation in the graph executes exactly once, so every value can be assigned a unique 

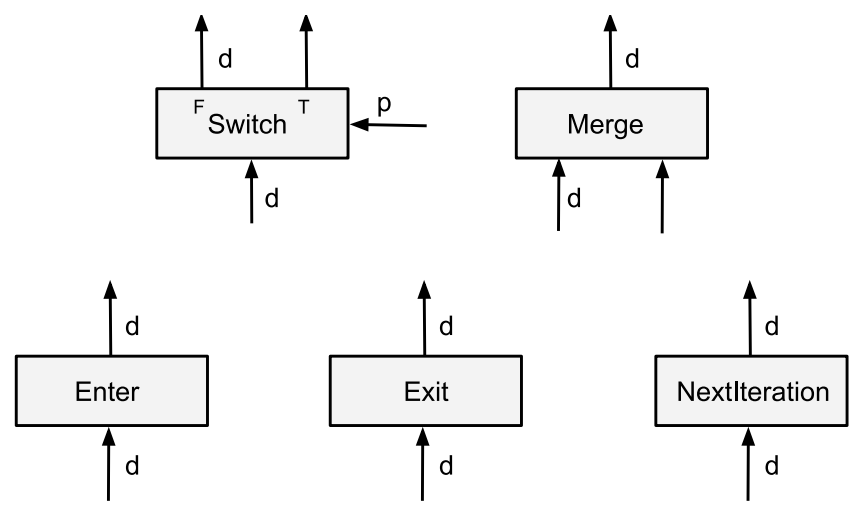

Figure 3: The control-flow primitives.

name, and every Send/Recv pair a unique rendezvous key at graphconstruction time. With control flow, this property no longer holds: an operation in a loop can execute zero or more times, and therefore the unique names and rendezvous keys must be generated dynamically to distinguish multiple invocations of the same operations.

One attractive feature of TensorFlow is that it imposes no restrictions on partitioning: an operation can be assigned to a device provided the device has the capability to run the corresponding computation, independently of graph topology or other considerations. By design, our control-flow extensions preserve this important feature. Therefore, a conditional branch or a loop body can be arbitrarily partitioned to run across many devices. For conditional computation, we must provide a mechanism to inform any waiting Recv operations on untaken branches, to reclaim resources. Similarly, for iterative computation, we must provide a mechanism to allow a loop-participating partition to start its next iteration or terminate its computation. The next section explains how we realize these behaviors.

TensorFlow does not aspire to provide fine-grained fault tolerance, and the iterative programs that use our mechanism typically run to completion between coarse-grained checkpoints. Because the typical duration of a computation using our control-flow constructs is much shorter than the expected mean time between failures, we rely on TensorFlow's coarse-grained checkpointing mechanism without changes.

\section{DESIGN AND IMPLEMENTATION}

We rely on a small set of flexible, expressive primitives that serve as a compilation target for high-level control-flow constructs within a dataflow model of computation. We explain these primitives in Section 4.1. In Section 4.2, we then describe how we use these primitives for compilation. Finally, in Sections 4.3 and 4.4, we describe our design and implementation for local and distributed execution, respectively.

\subsection{Control-Flow Primitives}

Figure 3 shows our control-flow primitives namely Switch, Merge, Enter, NextIteration, and Exit. These primitives resemble those introduced in the classic dynamic dataflow machines developed by Dennis [13] and Arvind et al. [4, 5]. With these primitives, every execution of an operation takes place within a "frame"; concretely, here, frames are dynamically allocated execution contexts associated with each iteration of a loop. In TensorFlow without control flow, each operation in the graph executes exactly once; when extended with control flow, each operation executes at most once per frame.

The following is a brief description of the intended semantics.

- Switch takes a data input $d$ and a boolean input $p$, and forwards the data to one of its outputs $d_{f}$ or $d_{t}$, based on the value of predicate $p$.

- Merge forwards one of its available inputs $d_{1}$ or $d_{2}$ to its output; Merge is unlike other TensorFlow primitives in that it is enabled for execution when any of its inputs is available.

- Enter forwards its input to a child frame with a given name. There can be multiple Enter operations for the same child frame, each asynchronously making a different tensor available to the child frame. The child frame is created when the first Enter is executed in the runtime.

- Exit forwards a value computed in a frame to its parent frame. There can be multiple Exits to the parent frame, each asynchronously making a tensor available to the parent frame.

- NextIteration forwards its input to the next iteration's frame. Iteration $N+1$ begins when the first NextIteration operation is executed at iteration $N$. There can be multiple NextIterations in a frame.

\subsection{Compilation of Control-Flow Constructs}

We compile high-level control-flow constructs into dataflow graphs that comprise the primitives presented above. Next we outline the basics of the graph construction for conditionals and while-loops.

The translation of cond ( $p$, true_fn, false_fn) uses only Switch and Merge. We invoke true_fn and false_fn respectively to construct the two branches of a cond. During the construction of a branch, we capture any external tensor (not created in the branch), and insert a Swi tch to guard its entering into the branch. The guard ensures that any operations in a branch will be executed only when that branch is taken. The external tensors may become available at very different times; we use one Switch for each external tensor in order to maximize parallelism. Each branch may return several tensors, and both branches must return the same number and type of tensors. For each output, we add a Merge in order to merge the true and false branches, thus enabling downstream computation as soon as possible.

Figure 4 sketches the dataflow graph for a while-loop with a single loop variable. (If there were multiple loop variables, there would be a separate set of Enter, Merge, Switch, NextIteration, and Exit nodes for each of them, so that multiple iterations can run in parallel.) The loop predicate and the loop body are represented by the subgraphs $G_{\text {pred }}$ and $G_{\text {body }}$, respectively. The output of Merge is used as the input to $G_{\text {pred }}$ to compute the loop termination condition $p$, and as an input to Switch, which forwards the tensor either to Exit to terminate the current loop or to $G_{\text {body }}$ to start a new iteration. The graph is cyclic so the result of the loop body can go from one iteration to the next. Any external tensors used in $\mathrm{G}_{\text {pred }}$ or $G_{\text {body }}$ are captured and treated as loop constants. We automatically 


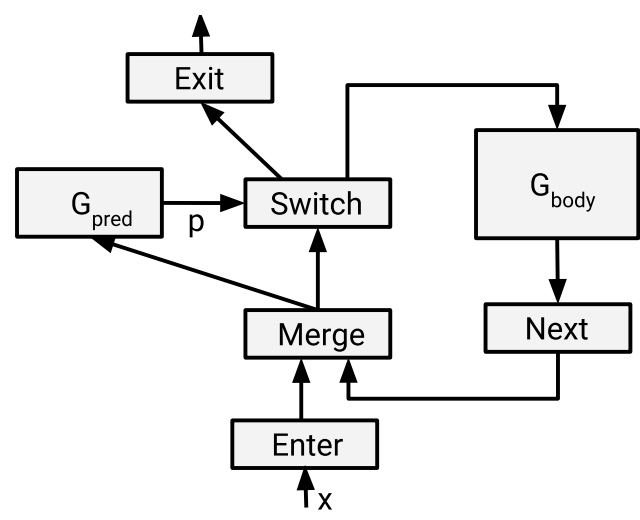

Figure 4: Dataflow graph for a while-loop.

insert an Enter for each external tensor to make it accessible in the loop body. When a new iteration is started, all the loop constants become available to that iteration automatically.

This approach supports arbitrary nestings of conditionals and loops. For example, a loop body can contain a conditional.

\subsection{Local Execution}

Recall that TensorFlow partitions a dataflow graph into a set of subgraphs. Each subgraph runs on a separate device, managed by a local executor that runs on the device's host. (The host's CPU is also represented as a device.) This subsection describes how such a local executor can support dynamic control flow.

The local executor is conceptually straightforward. It starts from the source nodes and repeatedly executes the nodes that become ready. A node, with the exception of Merge, becomes ready when all its inputs are available. All Recv nodes in a subgraph are regarded as source nodes.

For graphs without control-flow constructs, every node is executed exactly once and the execution ends when all nodes have been executed. Dynamic control flow introduces new complexity. An operation now can be executed any number of times. The executor must manage the (possibly concurrent) execution of multiple instances of the same operation, and to determine the completion of the entire execution.

We redesigned the local executor of TensorFlow to handle dynamic control flow. In order to track the tensors generated by different invocations of the same operation, each tensor inside the executor is represented as a tuple (value, is_dead,tag), where value is a tensor value, is_dead is a boolean that indicates whether the tensor is on an untaken branch of a Switch, and tag is a globally unique identifier for the tensor. Intuitively, the tag defines a dynamic execution context-a frame, in the terminology of Section 4.1. Each loop iteration starts a new frame, and within a frame an operation is executed at most once. As a result, the tag is used to distinguish the tensors generated by different iterations. This distinction is critical for the correct rendezvous of Send and Recv operations, since tags are used as the rendezvous keys.

The executor implements the evaluation rules shown in Figure 5. Each evaluation rule $\operatorname{Eval}(e, c)=r$ describes how to evaluate expression e in frame $c$, yielding output $r$. The operations Enter

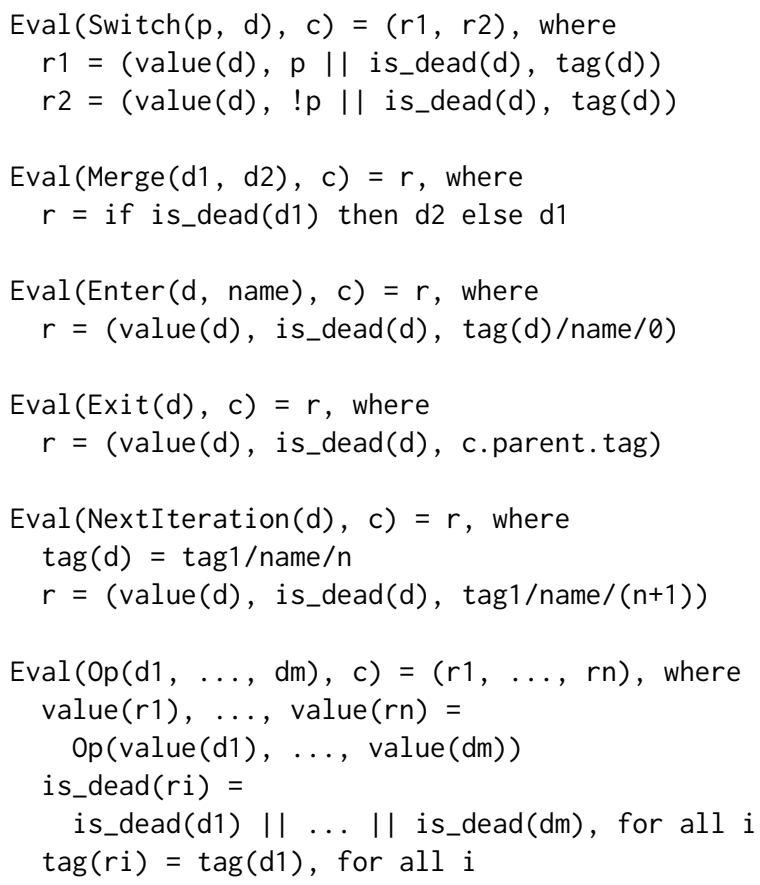

Figure 5: Evaluation rules for control-flow operators.

and Exit create and delete execution frames, respectively; for simplicity, the rules do not show this. All the inputs to an operation must have the same matching tag; c. parent is c's parent frame, with c. parent. tag as its tag.

The last rule applies to all non-control-flow operations. In the implementation, the actual computation is performed only when none of the inputs are dead. If there is a dead input, we skip the computation and propagate a dead signal downstream. This propagation of deadness is useful for supporting distributed execution, as explained in the next subsection. The choice to propagate the tag of the first input is arbitrary; all inputs must have the same tag.

While these rules allow multiple loop iterations to run in parallel, more parallelism typically results in more memory consumption. We therefore incorporate knobs in the local executor that allow us to limit the degree of parallelism. In our evaluation, we generally find that a limit of 32 works well-better than 1, which would be easy to achieve with stricter implementation strategies, but also better than no limit at all. The optimal value depends on the details of the hardware and the model.

\subsection{Distributed Execution}

The main challenge for distributed, dynamic control flow arises when the subgraph of a conditional branch or loop body is partitioned across devices. We favor a design that allows the executors of the partitions to make progress independently, with no centralized coordinator. We do not require synchronization after loop iterations, as this would severely limit parallelism. Each device that participates in a loop can start the next iteration or exit once it receives the value of the loop predicate. A partition can have multiple iterations running in parallel, and partitions can work on different 

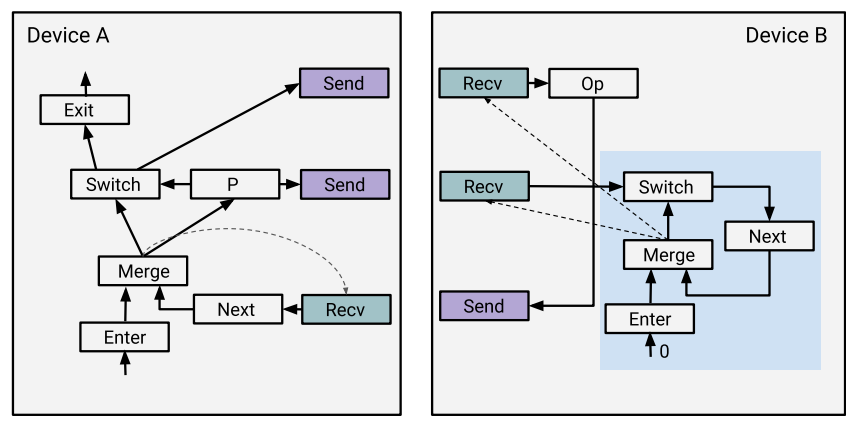

Figure 6: Distributed execution of a while-loop.

iterations of the same loop. The local executors communicate only via Send and Recv operations. A centralized coordinator is involved only in the event of completion or failure.

We first look at conditionals, and consider a Recv operation on an untaken branch. A Recv is always ready and can be started unconditionally. So the system would block, without reclaiming resources, if the corresponding Send is never executed. The solution is to propagate an is_dead signal across devices from Send to Recv. The propagation may continue on any number of devices. This propagation scheme handles distributed execution of nested conditionals, and interacts well with distributed execution of loops. However, when there are many Send-Recv pairs across devices on a rarely taken conditional branch, the large number of is_dead signals may cause performance issues. These situations seldom arise, but we have prototyped an optimization that transmits an is_dead signal only once to the destination device and, there, broadcasts it to all Recv operations.

For the distributed execution of a loop, at each iteration, each partition needs to know whether to proceed or exit. We address this need by automatically rewriting the graph with simple controlloop state machines. For example, Figure 6 shows the result of partitioning a simple while-loop across two devices. The loop body contains only one operation Op assigned to device B. In B's partition we add a control-loop state machine (blue shade in the figure), which controls the Recv operations inside the loop. The dotted lines are control edges, and impose an order on operations. Suppose that device $\mathrm{A}$ is executing the loop predicate $\mathrm{P}$ at iteration $i$; distributed execution may then proceed as follows:

- On A, Recv awaits a value from B. A sends P to B, so B knows the decision on iteration $i$ and, depending on whether $\mathrm{P}$ is true, A sends either the input tensor for Op or a dead signal to $\mathrm{B}$.

- On B, if Recv for Op gets a real tensor from A, B executes $O p$ and sends back a real tensor. Otherwise, if the Recv gets an is_dead signal, B propagates the signal through Op and sends an is_dead signal back to A. If Recv for Switch gets true, the control-loop state machine further enables Recvs for the next iteration. Otherwise, the control loop terminates, and so does execution on B.

- Back on A, if Recv gets a real tensor, the next iteration is started. Otherwise, execution terminates.
The overhead for the distributed execution of a loop is that every participating device needs to receive a boolean at each iteration from the device that produces the loop predicate. However, the communication is asynchronous and computation of the loop predicate can often run ahead of the rest of the computation. Given typical neural network models, this overhead is minimal and largely hidden.

To make things concrete, let us take a brief look at GPU execution. In this setting, the compute and I/O operations typically run on GPU as a sequence of asynchronous kernels, whereas control-flow decisions are made by the local executor for the GPU on the host. From the point of view of this local executor, a GPU kernel is considered completed once it is enqueued into the correct GPU stream. (Correctness is guaranteed by the sequential execution of the kernels in a single stream and proper cross-stream synchronization.) So once we allow parallel iterations, the local executor will typically run completely in parallel to the compute and I/O operations and on separate computing resources. Therefore, as we will show in Section 6, dynamic control flow gives the same performance as static unrolling.

\section{AUTOMATIC DIFFERENTIATION AND MEMORY MANAGEMENT}

Machine learning algorithms often rely on gradient-based methods for optimizing a set of parameters. During the training of models, gradient computations usually take more than half of the compute time. It is therefore critical to make these computations efficient and scalable.

TensorFlow supports automatic differentiation: given a dataflow graph that represents a neural network, TensorFlow generates efficient code for the corresponding distributed gradient computations. This section describes how we extend automatic differentiation to control-flow constructs, and (briefly) the treatment of TensorArrays.

This section also describes techniques for memory management. Although these techniques are motivated by automatic differentiation, they are not specific to this purpose.

\subsection{Backpropagation with Control Flow}

TensorFlow includes a reverse-mode automatic differentiation (autodiff) library that implements the well-known backpropagation algorithm [35], and we describe here its support for (potentially nested) cond and while_loop constructs.

We first revisit the basic autodiff algorithm used in TensorFlow. The $t f$.gradients () function computes the gradients of a scalar function, $f\left(x_{1}, x_{2}, \ldots\right)$, with respect to a set of parameter tensors, $x_{1}, x_{2}, \ldots$, using the following algorithm, which implements the vector chain rule: ${ }^{1}$

(1) Identify the subgraph $G$ of operations between the symbolic tensor representing $y=f\left(x_{1}, x_{2}, \ldots\right)$ and each parameter tensor $x_{1}, x_{2}, \ldots$.

(2) For each edge in $G$, which represents an intermediate value $t$ in $f$, set $\operatorname{Grads}[t]:=0$. Set $\operatorname{Grads}[y]:=1$.

\footnotetext{
${ }^{1}$ Parr and Howard survey the necessary calculus to understand this algorithm [31].
} 


\section{Forward}

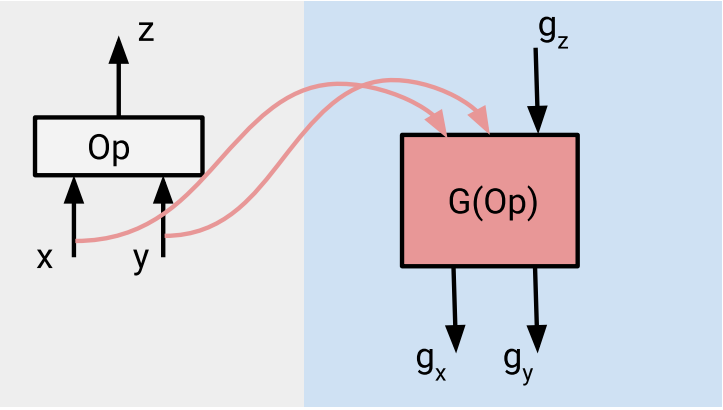

Figure 7: An operation and its gradient function.

(3) Traverse the vertices of $G$ in reverse topological order. For each vertex representing an intermediate operation

$$
o_{1}, \ldots, o_{n}=O p\left(i_{1}, \ldots, i_{m}\right),
$$

invoke the corresponding "gradient function"

$$
\partial i_{1}, \ldots, \partial i_{m}=\operatorname{OpGrad}\left(i_{1}, \ldots, i_{m}, \operatorname{Grads}\left[o_{1}\right], \ldots, \operatorname{Grads}\left[o_{n}\right]\right) \text {. }
$$

Add each $\partial i_{k}$ to Grads $\left[i_{k}\right]$.

(4) After traversing every vertex, the gradient $\frac{\partial y}{\partial x_{k}}$ is $\operatorname{Grads}\left[x_{k}\right]$.

TensorFlow includes a library of gradient functions that correspond to most of its primitive operations. Figure 7 shows a schematic example of such a gradient function, G(Op), which depends on both the partial derivative of $y$ with respect to the original Op's output $\left(g_{z}\right)$ and the original Op's inputs ( $x$ and $\left.y\right)$. As a concrete example, the $t f . m a t m u l(x, y)$ operation has the following (simplified) gradient function, which computes gradients with respect to matrices $x$ and $y$ :

\# NOTE: 'op' provides access to the inputs

\# and outputs of the original operation. def MatMulGrad(op, g_z):

$x, y=$ op.inputs

$g_{-} x=t f . m a t m u l\left(g_{-} z\right.$, tf.transpose $\left.(y)\right)$

$g_{-} y=t f . m a t m u l\left(t f . t r a n s p o s e(x), g_{-} z\right)$

return g_x, g_y

Tensors $\mathrm{x}$ and $\mathrm{y}$ are used in the gradient function, so will be kept in memory until the gradient computation is performed. The resulting memory consumption is a major constraint on our ability to train deep neural networks, as we need values from all layers of the forward computation (that is, of the computation being differentiated) for the gradient computation. The same difficulty applies to neural networks with loops. We return to this topic in Section 5.3.

We describe here the mechanisms that enable TensorFlow's autodiff algorithm to perform backpropagation through control-flow constructs. Each operation in the graph is associated with a "controlflow context" that identifies the innermost control-flow construct of which that operation is a member. When the backpropagation traversal first encounters a new control-flow context, it generates a corresponding control-flow construct in the gradient graph.

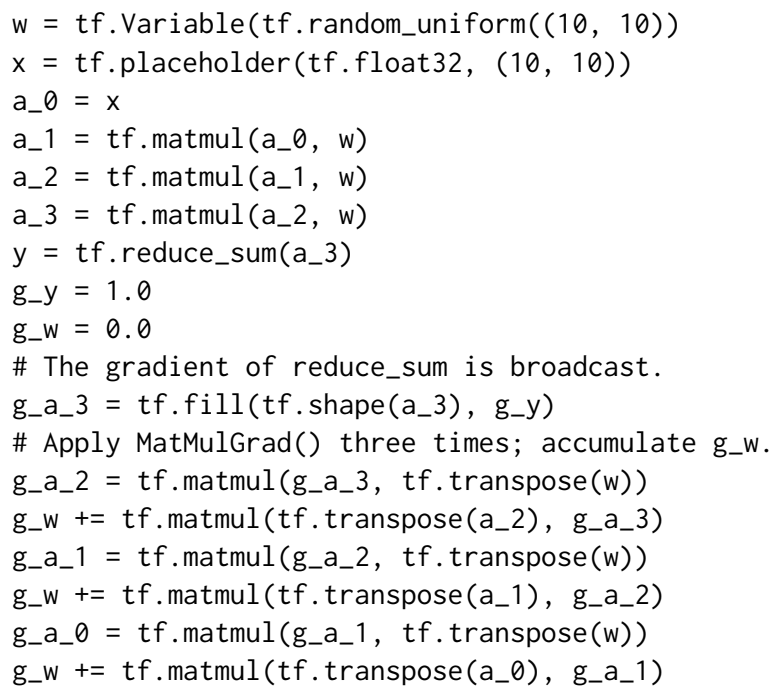

Figure 8: Computing the gradient of a loop by unrolling.

The gradient for $t f$.cond(pred, true_fn, false_fn) with output gradients $g \_z$ is

$t f$. cond (pred, true_fn_grad(g_z), false_fn_grad(g_z)).

To obtain the true_fn_grad function, we apply tf.gradients() to the symbolic outputs $t_{i}$ of true_fn, but setting the initial gradients Grads $\left[t_{i}\right]:=$ g_z $[i]$; the same logic applies to false_fn_grad.

The logic for tf. while_loop(cond, body, loop_vars) is more complicated. To understand its key components, consider the following simple program that iteratively multiplies an input matrix $x$ by a parameter matrix $w$ :

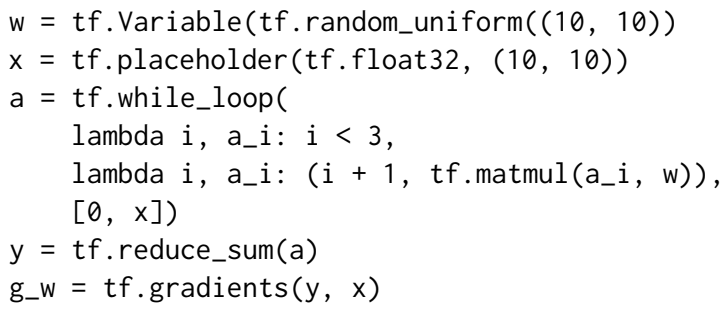

In this simple example, we can take advantage of the loop bound being a constant. Figure 8 illustrates how we might unroll the loop statically and apply the gradient functions for $t f$.matmul() and tf. reduce_sum() $:^{2}$

This example highlights three features of the general solution:

(1) The gradient of a tf.while_loop() is another loop that executes the gradient of the loop body for the same number of iterations as the forward loop, but in reverse. In general, this number is not known statically, so the forward loop must be augmented with a loop counter.

(2) The gradient of each differentiable loop variable becomes a loop variable in the gradient loop. Its initial value is the

\footnotetext{
${ }^{2}$ In the general case, the loop bound may depend on the input data-e.g., based on the length of a sequence in an $\mathrm{RNN}$-and we must construct a tf. while_loop() for the gradients.
} 


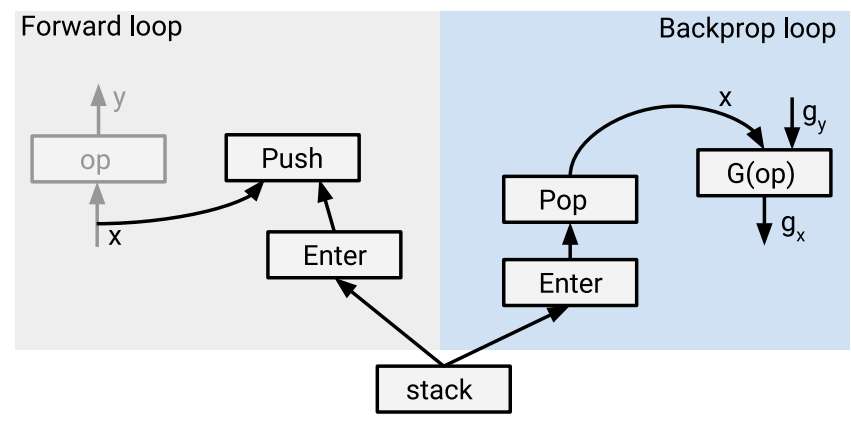

Figure 9: Saving a tensor for reuse in backpropagation.

gradient with respect to the corresponding loop output (e.g., g_a_3 in the example).

(3) The gradient of each differentiable tensor that is constant in the loop (e.g., g_w in the example) is the sum of the gradients for that tensor at each iteration.

In addition, intermediate values a_1, a_2, and a_3 from the forward loop are used in the gradient loop. The performance of our solution depends heavily on how we treat such intermediate values. There are typically many such values, such as the inputs of a matrix multiplication or the predicate of a conditional nested in the loop. We avoid the computational expense of recomputing these values by automatically rewriting the forward loop to save any intermediate values that the gradient loop needs. We introduced a stack data structure into TensorFlow to save values across loops: the forward computation pushes onto the stacks, the gradient computation pops. Figure 9 shows the graph structure that implements this stack-based state saving. The implementation uses a different stack for each intermediate value that is reused in the gradient loop, in order to allow individual gradients to be computed asynchronously. We prefer to use a regular tensor to store each intermediate value, because each intermediate value might have a different shape, and packing the values into a dense, contiguous array would incur unnecessary memory copies. However, if the loop variables have a static shape and the iteration count has a static upper bound, the XLA [46] compiler may lower the stack operations to read/write operations on a contiguous mutable array.

Correctness requires care to preserve the proper ordering of stack operations. Performance considerations lead us to making the stack operations asynchronous, so that they can run in parallel with the actual computation. For example, in Figure 9, Op (and even operations in subsequent iterations) can potentially run in parallel with Push. As Section 5.3 explains, this asynchrony is important for overlapping compute and I/O operations. For correctness, we add explicit control dependencies to enforce ordering of the stack operations.

Our implementation has additional optimizations, often with the goal of reducing memory usage. For example, if a value is immediately reduced in the gradient code by an operation that computes its shape, rank, or size, we move this operation into the forward loop. Moreover, for calculations that accumulate gradients, we introduce subgraphs that sum gradients eagerly into new loop variables.
Our approach also accommodates nested control-flow constructs. When a conditional nests inside a while-loop, we push the guard values at all forward iterations onto a stack, and pop those values to control the conditionals in the gradient loop. For nested loops, we apply our techniques recursively. Although we hope this explanation gives the intuition for control-flow operator gradients, a rigorous mathematical treatment is beyond the scope of this paper. The literature on automatic differentiation has considered the question of correctness of the semantics of control flow with respect to the mathematical notion of differentiation (e.g., [8]), and our algorithms follow these established principles. That said, this question remains a subject of ongoing research [33].

\subsection{Backpropagation with TensorArrays}

TensorArrays constitute an important element of our programming model, so automatic differentiation must treat them correctly and efficiently. For this purpose, we require that each location of a TensorArray may be written only once in the forward computation being differentiated, but allow multiple reads from the same location. This requirement is satisfied by common applications of TensorArrays.

The TensorFlow runtime represents TensorArrays as "resource objects", which are containers for mutable state. Each TensorArray ta exposes an opaque handle ta. handle. Operations such as write and read accept a TensorArray handle as their primary argument. During backpropagation, for each forward TensorArray a new TensorArray of the same size is created to hold gradient values. The operation ta.grad(), when executed, either creates or performs a table lookup for the gradient TensorArray associated with handle ta. handle. The TensorArray operations are duals of each other: the gradient of ta.read(ix) is ta. $\operatorname{grad}()$. write(ix, $\left.g_{i x}\right)$, and vice versa, and the gradient of ta. unstack(ts) is ta. $\operatorname{grad}() \cdot \operatorname{stack}()$, and vice versa. When there are multiple reads to the same location, the gradient TensorArray holds the sum of the partial gradients generated by the reads. Our implementation ensures the proper ordering of reads and writes while allowing parallelism.

\subsection{Memory Management}

The memory demands described above are critical on specialized devices such as GPUs, where memory is typically limited to no more than $16 \mathrm{~GB}$. We employ several techniques for alleviating this scarcity. We rely on memory swapping, taking advantage of temporal locality. We move tensors from GPU to CPU memory (which is relatively abundant) when they are pushed onto stacks, and bring them back gradually before they are needed in backpropagation. The key to achieving good performance in memory swapping is to overlap compute and I/O operations. This goal requires the seamless cooperation of several system components.

First, as explained above, multiple iterations of a loop can run in parallel, and the stack push and pop operations are asynchronous and can run in parallel with the computation proper. The forward computation can run ahead without waiting for the completion of I/O operations. Conversely, during the gradient computation, the I/O operations can run ahead, prefetching the tensors that will be needed next. 


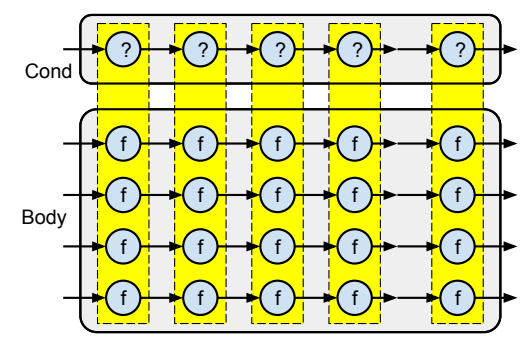

(a) Independent devices

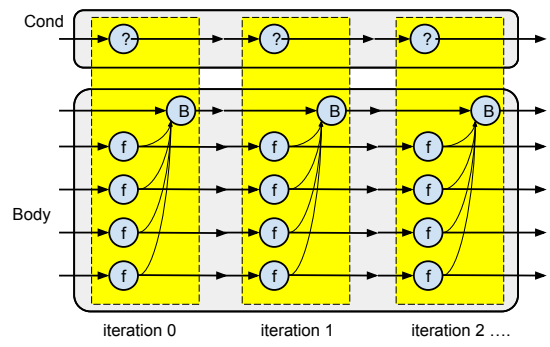

(b) Barrier / AllReduce

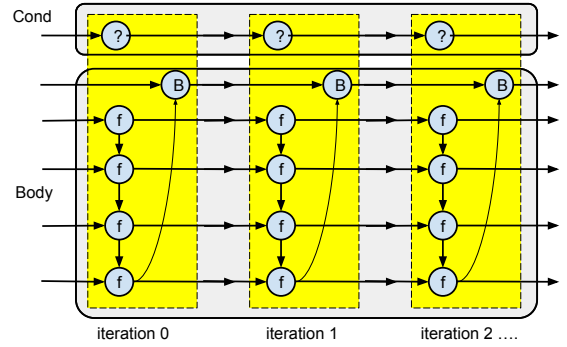

(c) Data-dependent loop body

Figure 10: Dataflow dependencies in a distributed while-loop.

Second, in the context of GPUs, we use separate GPU streams for compute and I/O operations to further improve the overlap of these two classes of operations. Each stream consists of a sequence of GPU kernels that are executed sequentially. Kernels on different streams can run in parallel with respect to each other. We therefore use (at least) three streams for compute, CPU-to-GPU transfer, and GPU-to-CPU transfer operations, respectively. Thus, we run compute kernels in parallel with independent memory-transfer kernels. Of course, special care must be taken when there is a causal dependency between two kernels on different streams. We rely on a combination of TensorFlow control edges and GPU hardware events to synchronize the dependent operations executed on different streams.

Our implementation of the scheme described above watches the memory consumption reported by the TensorFlow memory allocator, and only starts to swap when memory consumption reaches a predefined threshold. We also do not swap small tensors or the same value more than once.

This scheme has a large impact. For instance, as Section 6 describes, for an example RNN model for processing sequences, without memory swapping, we run out of memory for sequences of length 500. With memory swapping, we can handle sequences of length 1000 with little overhead, and available host memory is the primary factor limiting the maximum sequence length.

\section{EVALUATION}

In this section, we evaluate our design and implementation, focusing on key design choices, and comparing against performance baselines. In particular, our main comparisons against TensorFlow without in-graph dynamic control flow are Table 1 (comparing with the system with swapping disabled), Figure 14 (comparing with static unrolling), and Section 6.5 (comparing with out-of-graph control flow). Other experiments (in particular Figures 12 and 15) give evidence of the performance benefits of our approach relative to more simplistic approaches that would limit parallelism or distribution. We focus on these baselines because they permit the most direct, apples-to-apples comparison.

For all the experiments, unless otherwise stated, we run TensorFlow on a shared production cluster, consisting of Intel servers with NVidia Tesla K40 GPUs connected by Ethernet across a production networking fabric, and reported performance numbers are averages across five or more repeated runs.

\subsection{Data Dependencies}

In this experiment we use simple microbenchmarks to evaluate the performance and scalability of distributed iterative computation. The benchmark is a single while-loop with its loop body partitioned to run on a cluster of GPUs. First we consider two common patterns of data dependence for the loop body: one where there is no coordination between devices, and one where devices synchronize at the end of each iteration using a barrier (e.g., AllReduce), as illustrated in Figures 10(a) and 10(b). Such computation patterns are quite common in multi-layer architectures with MoEs and RNNs.

We evaluate the overall system capacity in terms of the number of iterations per second it can support when running a while-loop distributed across a set of GPUs. Each GPU is hosted on a separate machine in the cluster. The computation $f$ on each GPU is a very small matrix operation, optionally followed by a barrier $B$ across all GPUs, so this experiment gives us the maximum number of distributed iterations the system can handle at various scales.

Figure 11 shows the number of iterations achieved per second as we vary the number of machines from 1 to 64 . We plot the median and 5 th/95th percentile performance from 5000 trials. When the loop body has no cross-device dependencies, the system can support over 20,000 iterations per second on a single machine, decreasing to 2014 with 64 machines. (i.e., $457 \mu$ s per iteration). If the loop contains a barrier operation, this reduces to 809 iterations per second $(1235 \mu \mathrm{s}$ per iteration).

Figure 11 demonstrates that, for both patterns of data dependency, the overhead of distributed execution remains acceptable as the number of machines increases. The Barrier result is within a factor of two of the global barrier scaling results of Naiad [27, Figure 6(b)], although the variance here is much lower. Other experiments (in particular, those in Figure 15) further characterize the scaling that results from distributed execution using non-synthetic workloads.

Next we evaluate the benefits of running multiple iterations in parallel. We run our simple benchmark on 8 GPUs in a single server and vary the number of parallel iterations from 1 to 32 . The loop consists of 8 layers of computation, one for each GPU; and each GPU performs a $1024 \times 1024$ matrix multiplication before passing the result of its computation to the next. Each GPU has a data dependency on its state from the previous loop iteration, and on the output of the previous GPU. The loop iterations are additionally serialized as shown in Figure 10(c). Note that the loop condition has 


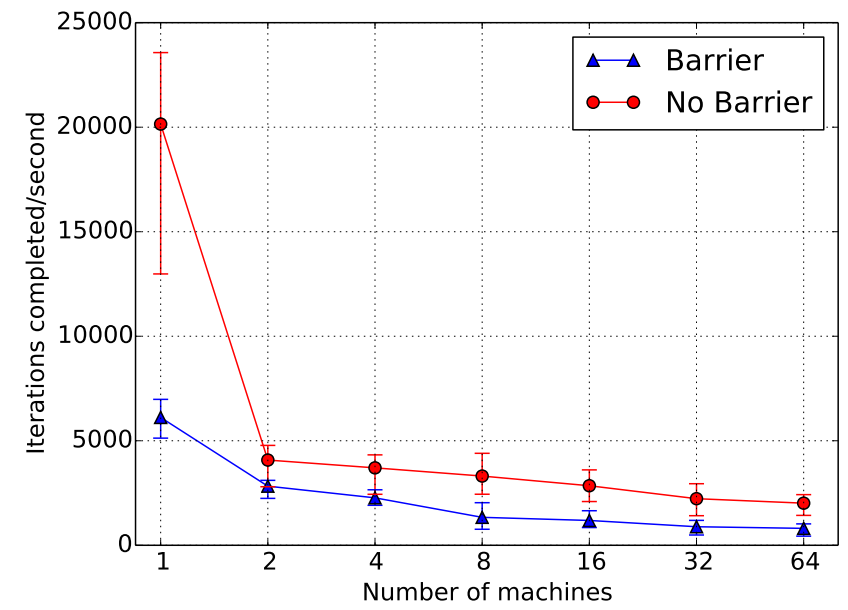

Figure 11: Performance of a distributed while-loop with a trivial body on a GPU cluster.

no data dependency on the body-when computing on GPUs, this independence can often allow CUDA kernels from many iterations to be enqueued on a stream for future execution.

We measure performance both on a K40 equipped server, as in the previous experiment, and on NVidia's flagship DGX-1 machine equipped with 8 V100 GPUs, plotting the median and 5th/90th percentiles from 5000 trials.

Figure 12 demonstrates that running iterations in parallel is crucial for achieving the inherent parallelism from the 8 GPUs; computation is automatically pipelined so as to mask any data dependencies. On the K40 machine, we reach the peak performance when the setting for parallel iterations is above 8. On the faster V100 server we achieve highest performance with 4 parallel iterations, but additional parallelism introduces scheduling noise. This experiment also gives us a comparison with out-of-graph loop execution. When the parallel iteration count is set to 1 , loop iterations are executed sequentially, similarly to straightforward out-of-graph execution driven by a single thread. As Figure 12 indicates, in-graph control flow makes it easy to exploit parallelism, here giving 5 times more iterations per second than the unparallelized approach.

\subsection{Memory Management}

In this experiment we evaluate the effectiveness of swapping memory between CPU and GPU. TensorFlow's RNN implementation (known as dynamic_rnn) is based on our work, and has been used in a wide variety of machine learning applications, including Neural Machine Translation (see Section 2). We use dynamic_rnn in our experiments. The model architecture in this experiment is a single-layer LSTM [19] with 512 units. The RNN implementation is available in the current distribution of TensorFlow [42].

One key measure for RNN performance is the ability to train on long sequences with large batch sizes. Long sequences are important in many applications; examples include patient history in health care, view history in web sites that recommend content, and signal sequences in speech or music. The use of sufficiently large batch sizes is crucial for efficiency on devices such as GPUs. Table 1 shows

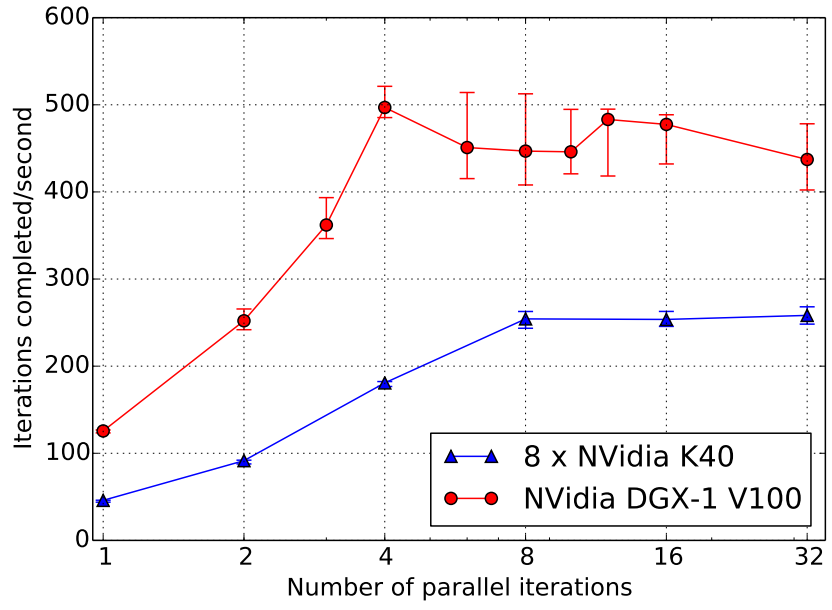

Figure 12: Effect of changing the number of iterations that are allowed to run concurrently.

the performance of training as we increase the sequence length from 100 to 1000 . All results are for a single GPU with batch size 512. When memory swapping is disabled, we run out of memory (OOM) at sequences of length a little over 500 . When memory swapping is enabled, we can train on sequences of length 1000 with no overhead. This increase in sequence length allows users to train substantially larger and deeper networks (e.g., with multiple LSTM layers) at no additional cost. For those models, the maximum length of a sequence whose state fits in GPU memory would decrease even further (e.g., to 500/8 for an 8-layer LSTM model) in the absence of optimizations such as swapping or model parallelism (which we discuss in Section 6.4).

We attribute this scalability to the ability to overlap compute operations with the I/O operations for memory swapping. This ability arises from the combination of parallel iterations, multistream asynchronous GPU kernel execution, and asynchronous state saving in gradient computations ( $§ 5.3)$. Figure 13 shows the timelines for the kernels executed on both the compute and I/O streams of the GPU. The kernels on the compute stream (labeled as Compute) are the compute operations of the LSTMs; the kernels on the two I/O MemCpy streams (labeled as DtoH and HtoD) are copy kernels that transfer tensors between CPU and GPU. The figure shows a time window from the forward computation, so the GPU-to-CPU stream (DtoH) is active and the CPU-to-GPU stream (HtoD) is mostly idle. As the figure indicates, the execution of the compute kernels and the I/O kernels proceed in parallel, so the total elapsed time with memory swapping is almost identical to the elapsed time without it.

\begin{tabular}{c|rrrrrrr} 
& \multicolumn{8}{|c}{ Training time per loop iteration (ms), by sequence length } \\
\cline { 2 - 7 } Swap & 100 & 200 & 500 & 600 & 700 & 900 & 1000 \\
\hline Disabled & 5.81 & 5.78 & 5.75 & OOM & OOM & OOM & OOM \\
Enabled & 5.76 & 5.76 & 5.73 & 5.72 & 5.77 & 5.74 & 5.74
\end{tabular}

Table 1: Training time per loop iteration for an LSTM model with increasing sequence lengths. 


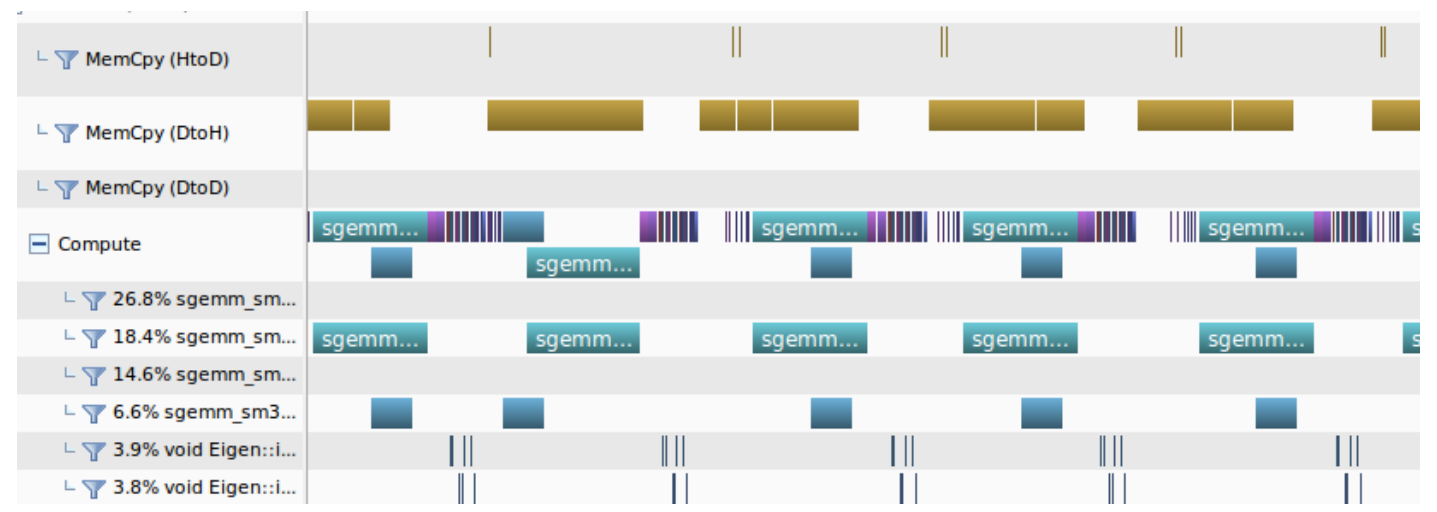

Figure 13: Timelines for the GPU kernels with memory swapping enabled.

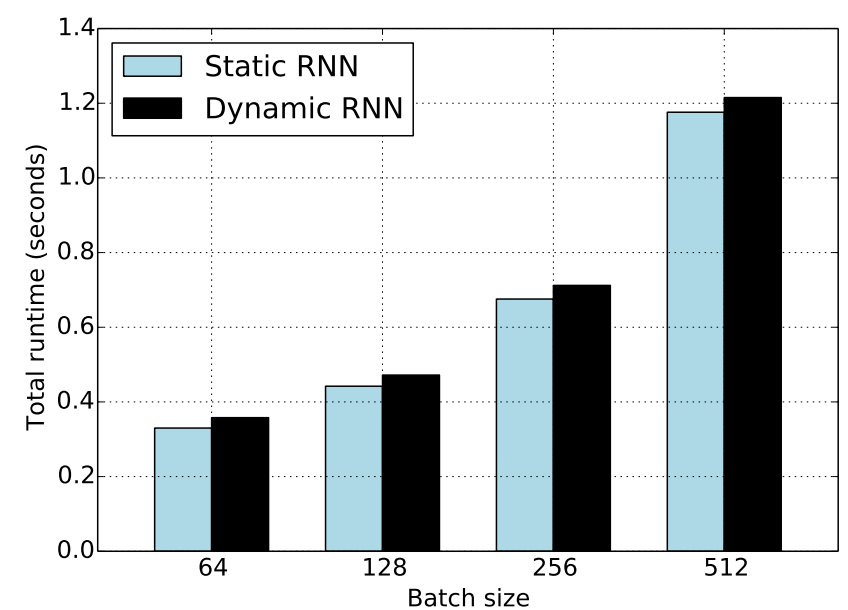

Figure 14: Performance comparison of dynamic control flow against static unrolling.

\subsection{Dynamic Control Flow vs. Static Unrolling}

An alternative to using dynamic_rnn is to rely on static loop unrolling. Static unrolling eliminates dynamic control flow and therefore gives a good baseline in understanding the performance of dynamic control flow.

Figure 14 shows the total elapsed times of running one training step with various batch sizes. All results are for a single-layer LSTM running on one GPU with sequence length 200 . We see a small slowdown of between $3 \%$ and $8 \%$, and the slowdown decreases as we increase the batch size (and hence the computation). The slowdown is largely due to the overhead of dynamic control flow.

We also consider the memory consumption of dynamic_rnn against static unrolling. In some configurations, dynamic_rnn can handle substantially longer sequences than static unrolling. For example, for a single layer LSTM model of 2048 units and batch size 256, dynamic_rnn can handle sequences of length 256 while static unrolling runs out of memory at 128 . Static unrolling exposes the entire unrolled dataflow graph (and hence all the potential

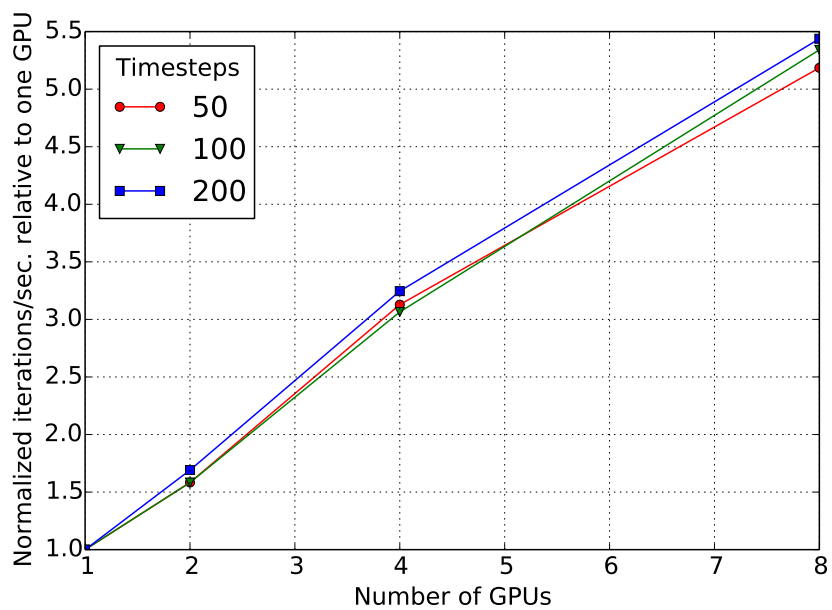

Figure 15: Parallel speedup for an 8-layer LSTM as we vary the number of GPUs from 1 to 8 .

parallelism) to the runtime. The abundant parallelism poses a challenge to the runtime as the order of the operations can dramatically impact memory usage. With dynamic control flow, the additional structure enables the runtime to choose an execution order that is both time- and memory-efficient.

\subsection{Model Parallelism}

For several reasons such as better memory utilization, users often want to train models in parallel across multiple devices. For example, a multi-layer RNN can be parallelized across multiple GPUs by assigning each layer to a different GPU. In our approach, this strategy is expressed as a single loop that is partitioned across GPUs. Recall that in Figure 12, we show how a microbenchmark performs in such a setting. We now evaluate the performance of an end-to-end training step of a realistic 8-layer LSTM model on 8 GPUs. Figure 15 shows the total elapsed time as we vary the number of GPUs. We observe a parallel speedup from 1 to 8 GPUs, and a speedup of $5.5 \times$ at 8 GPUs. As expected, the speedup is sub-linear, due to the additional DMA overhead when using multiple GPUs, 


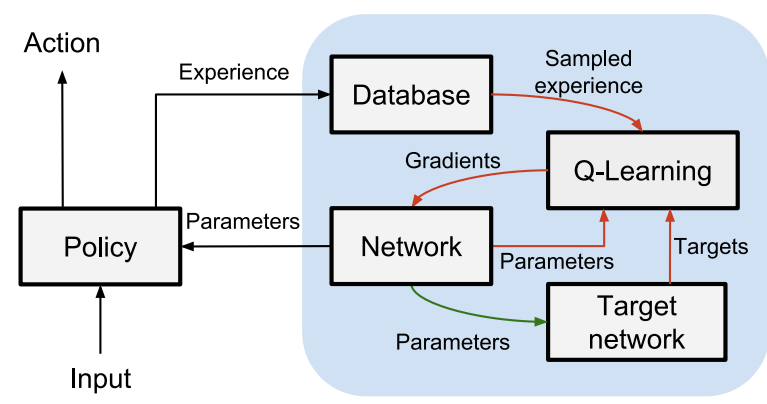

Figure 16: Dynamic control flow in Deep Q-Networks.

but this is mitigated by the ability to overlap computation in multiple iterations. The running time includes the gradient computation, so this experiment additionally illustrates the performance of the parallel and distributed execution of gradient computations.

\subsection{An Application: Reinforcement Learning}

Finally, we consider the benefits of dynamic control flow in an archetypical application: we describe how Deep Q-Networks (DQN) [26], a benchmark reinforcement learning algorithm, can be implemented with dynamic control flow. While DQN has already been superseded by newer methods, this example is representative of uses of dynamic control flow in reinforcement learning, including more recent algorithms.

Figure 16 shows a diagram of the DQN algorithm. DQN augments a neural network with a database in which it stores its incoming experiences, periodically sampling past experiences to be employed for Q-Learning, a form of reinforcement learning. Q-Learning uses these experiences along with a second neural network, called the target network, to train the main network. The target network is periodically updated to reflect a snapshot of the main network.

The DQN algorithm includes many conditionals: different experiences cause different types of writes to the database, and sampling of experiences, Q-Learning, and updates to the target network are each performed conditionally based on the total amount of experience received.

A baseline implementation of DQN without dynamic control flow requires conditional execution to be driven sequentially from the client program. The in-graph approach fuses all steps of the DQN algorithm into a single dataflow graph with dynamic control flow, which is invoked once per interaction with the reinforcement learning environment. Thus, this approach allows the entire computation to stay inside the system runtime, and enables parallel execution, including the overlapping of $\mathrm{I} / \mathrm{O}$ with other work on a GPU. It yields a speedup of $21 \%$ over the baseline. Qualitatively, users report that the in-graph approach yields a more self-contained and deployable DQN implementation; the algorithm is encapsulated in the dataflow graph, rather than split between the dataflow graph and code in the host language.

\section{RELATED WORK}

Our approach to control flow draws on a long line of research on dynamic dataflow architectures, going back to the work of Arvind et al. $[4,5]$. The timely dataflow model [28], implemented in the Naiad system [27], can be seen as a recent embodiment of those architectures. It supports distributed execution, with protocols for tracking the progress of computations. The control-loop state machines we describe in Section 4.4 constitute a specialized approach for this tracking that is more lightweight and efficient for the task; this approach, although suitable for our purposes, would be difficult to extend to incremental computations of the kind that Naiad enables. Naiad does not support heterogeneous systems, so does not address some of the problems that we studied, in particular memory management across heterogenous devices. Nor does it address automatic differentiation, which is crucial for machine learning applications.

Some systems for machine learning, such as Theano [6, 9] and CNTK [36], allow the programmer to create a computation graph, for example with a Python front-end, and then to launch the execution of this graph, following the in-graph approach described in the introduction. Theano allows this graph to contain control-flow constructs, but Theano's support for control flow is relatively limited. In particular, Theano allows neither nested loops, nor the parallel or distributed execution of control-flow constructs; its automatic differentiation often requires more computation and is therefore less efficient than the approach in this paper. In a discussion of limitations and challenges related to control flow, the developers of Theano have written that they find TensorFlow's approach appealing [2].

Other systems for machine learning, such as Torch [12], Chainer [44], and PyTorch [34], blur this phase distinction: a graph appears to be executed as it is defined, either on one input example at a time or on manually specified batches. Because the graph is not given ahead of time, optimizations are more difficult. These systems are typically based on Python, and expose Python's control-flow constructs, which do not come with support for distributed execution and memory management across heterogeneous devices. The two approaches are reconciled in systems such as MXNet [10, 14], which supports both (but without control flow in graphs), and with TensorFlow's "imperative mode" and "eager mode" extensions [23, 37].

While we favor embedding control flow in a static graph, others have proposed more dynamic distributed execution engines that support similar control flow. For example, CIEL represents a program as an unstructured "dynamic task graph" in which tasks can tail-recursively spawn other tasks, and imperative control-flow constructs are transformed into continuation-passing style [29]. Nishihara et al. recently described a system for "real-time machine learning" that builds on these ideas, and adds a decentralized and hierarchical scheduler to improve the latency of task dispatch [30]. Programming models based on dynamic task graphs are a direct fit for algorithms that make recursive traversals over dynamic data structures, such as parse trees [15]. By contrast, in our approach this recursion must be transformed into iteration, for example using the transformation that Looks et al. describe [24]. The drawback of an unstructured dynamic task graph is that the individual tasks are black boxes, and more challenging to optimize holistically.

The wish to save memory is fairly pervasive across machine learning systems, in part because of the important role of GPUs and other devices with memory systems of moderate size. Some techniques based on recomputation specifically target the memory 
requirements of backpropagation $[11,17]$. These techniques concern feed-forward graphs, LSTMs, and RNNs, rather than arbitrary graphs with control-flow constructs; for particular classes of graphs, clever algorithms yield efficient recomputation policies, sometimes optimal ones. Our work on swapping belongs in this line of research. So far, we have emphasized the development of mechanisms, and relatively simple but effective policies for their use. In future work we may explore additional algorithms and also the application of reinforcement learning to swapping and recomputation decisions.

Finally, our research is related to a substantial body of work on automatic differentiation (e.g., [18, 25, 32]). That work includes systems for automatic differentiation of "ordinary" programming languages (e.g., Fortran, C, or Python) with control-flow constructs. It has generally not been concerned with parallel and distributed implementations-perhaps because working efficiently on large datasets, as in deep learning, has not been a common goal [7].

\section{CONCLUSIONS}

This paper presents a programming model for machine learning that includes dynamic control flow, and an implementation of that model. This design enables parallel and distributed execution in heterogeneous environments. Automatic differentiation contributes to the usability of the control-flow constructs. The implementation allows us to leverage GPUs and custom ASICs with limited memory, and supports applications that make frequent control-flow decisions across many devices. Our code is part of TensorFlow; it has already been used widely, and has contributed to new advances in machine learning.

Dynamic control flow relates to active areas of machine learning, which may suggest opportunities for further work. In particular, conditional computation, where parts of a neural network are active on a per-example basis, has been proposed as a way to increase model capacity without a proportional increase in computation; recent research has demonstrated architectures with over 100 billion parameters [38]. Also, continuous training and inference may rely on streaming systems, perhaps using the dynamic dataflow architectures that we adapt, as suggested by work on timely dataflow and differential dataflow [28]. Further research on abstractions and implementation techniques for conditional and streaming computation seems worthwhile.

Dynamic control flow is an important part of bigger trends that we have begun to see in machine learning systems. Control-flow constructs contribute to the programmability of these systems, and enlarge the set of models that are practical to train using distributed resources. Going further, we envision that additional programminglanguage facilities will be beneficial. For instance, these may include abstraction mechanisms and support for user-defined data structures. The resulting design and implementation challenges are starting to become clear. New compilers and run-time systems, such as XLA [46], will undoubtedly play a role.

\section{ACKNOWLEDGMENTS}

We gratefully acknowledge contributions from our colleagues at Google and from members of the wider machine learning community, and the feedback that we have received from them and from the many users of TensorFlow. In particular, Skye WandermanMilne provided helpful comments on a draft of this paper. We also thank our shepherd, Peter Pietzuch, for his guidance in improving the paper.

\section{REFERENCES}

[1] Abadi, M., Barham, P., Chen, J., Chen, Z., Davis, A., Dean, J., Devin, M., Ghemawat, S., Irving, G., Isard, M., Kudlur, M., Levenberg, J., Monga, R., Moore, S., Murray, D. G., Steiner, B., Tucker, P., Vasudevan, V., Warden, P., Wicke, M., Yu, Y., AND Zheng, X. TensorFlow: A system for large-scale machine learning. In 12th USENIX Symposium on Operating Systems Design and Implementation (OSDI 16) (GA, 2016), USENIX Association, pp. 265-283.

[2] Al-Rfou, R., Alain, G., Almahairi, A., Angermueller, C., Bahdanau, D., Ballas, N., Bastien, F., Bayer, J., Belikov, A., Belopolsky, A., Bengio, Y., Bergeron, A., Bergstra, J., Bisson, V., Bleecher Snyder, J., Bouchard, N., Boulanger-Lewandowski, N., Bouthillier, X., de Brébisson, A., BreuleuX, O., Carrier, P.-L., Cho, K., Chorowski, J., Christiano, P., Cooijmans, T., Côté, M.-A., Côté, M., Courville, A., Dauphin, Y. N., Delalleau, O., Demouth, J., Desjardins, G., Dieleman, S., Dinh, L., Ducoffe, M., Dumoulin, V., Ebrahimi Kahou, S., Erhan, D., Fan, Z., Firat, O., Germain, M., Glorot, X., Goodfellow, I., Graham, M., Gulcehre, C., Hamel, P., Harlouchet, I., Heng, J.-P., Hidasi, B., Honari, S., Jain, A., Jean, S., Jia, K., Korobov, M., Kulkarni, V., Lamb, A., Lamblin, P., Larsen, E., Laurent, C., Lee, S., Lefrancois, S., Lemieux, S., LÉonard, N., Lin, Z., Livezey, J. A., Lorenz, C., Lowin, J., MA, Q., Manzagol, P.-A., Mastropietro, O., McGibbon, R. T., Memisevic, R., van MerriËnboer, B., Michalski, V., Mirza, M., Orlandi, A., Pal, C., Pascanu, R., Pezeshí, M., Raffel, C., Renshaw, D., Rocklin, M., Romero, A., Roth, M., Sadowski, P., Salvatier, J., Savard, F., Schlüter, J., Schulman, J., Schwartz, G., Serban, I. V., Serdyuk, D., Shabanian, S., Simon, E., Spieckermann, S., Subramanyam, S. R., Sygnowski, J., Tanguay, J., van Tulder, G., Turian, J., Urban, S., Vincent, P., Visin, F., de Vries, H., Warde-Farley, D., Webb, D. J., Willson, M., Xu, K., Xue, L., YAo, L., Zhang, S., And Zhang, Y. Theano: A Python framework for fast computation of mathematical expressions. arXiv preprint 1605.02688 (2016). arxiv.org/abs/1605.02688.

[3] Andor, D., Alberti, C., Weiss, D., Severyn, A., Presta, A., Ganchev, K., Petrov, S., AND Collins, M. Globally normalized transition-based neural networks. CoRR $a b s / 1603.06042(2016)$.

[4] Arvind, And Culler, D. E. Dataflow architectures. In Annual Review of Computer Science Vol. 1. Annual Reviews Inc., 1986, pp. 225-253. www.dtic.mil/cgibin/GetTRDoc?Location=U2\&doc=GetTRDoc.pdf \&AD=ADA166235.

[5] Arvind, ANd Nikhil, R. S. Executing a program on the MIT tagged-token dataflow architecture. IEEE Transactions on Computers 39, 3 (1990), 300-318. dl.acm.org/citation.cfm?id=78583.

[6] Bastien, F., Lamblin, P., Pascanu, R., Bergstra, J., Goodfellow, I. J., Bergeron, A., Bouchard, N., Warde-Farley, D., and Bengio, Y. Theano: new features and speed improvements. CoRR abs/1211.5590 (2012).

[7] Baydin, A. G., Pearlmutter, B. A., ANd Siskind, J. M. Tricks from deep learning. CoRR abs/1611.03777 (2016).

[8] Beck, T., AND Fischer, H. The if-problem in automatic differentiation. fournal of Computational and Applied Mathematics 50, 1-3 (1994), 119-131.

[9] Bergstra, J., Breuleux, O., Bastien, F., Lamblin, P., Pascanu, R., Desjardins, G., Turian, J., Warde-Farley, D., And Bengio, Y. Theano: A CPU and GPU math expression compiler. In Proceedings of the Python for scientific computing conference (SciPy) (2010), vol. 4, Austin, TX. UMontreal PDF.

[10] Chen, T., Li, M., Li, Y., Lin, M., Wang, N., Wang, M., XiAo, T., Xu, B., Zhang, C., AND ZHANG, Z. MXNet: A flexible and efficient machine learning library for heterogeneous distributed systems. In Proceedings of LearningSys (2015). www.cs.cmu.edu/ muli/file/mxnet-learning-sys.pdf.

[11] Chen, T., Xu, B., Zhang, C., And Guestrin, C. Training deep nets with sublinear memory cost. CoRR abs/1604.06174 (2016).

[12] Collobert, R., Bengio, S., And Mariéthoz, J. Torch: A modular machine learning software library. Tech. rep., IDIAP Research Institute, 2002. infoscience.epfl.ch/record/82802/files/rr02-46.pdf.

[13] Dennis, J. B., And Misunas, D. P. A preliminary architecture for a basic data-flow processor. In ACM SIGARCH Computer Architecture News (1975), vol. 3, ACM, pp. $126-132$.

[14] DMLC. MXNet for deep learning, 2016. github.com/dmlc/mxnet.

[15] Goller, C., AND KüChler, A. Learning task-dependent distributed representations by backpropagation through structure. IEEE Transactions on Neural Networks 1 (June 1996), 347-352.

[16] Graves, A. Adaptive computation time for recurrent neural networks. CoRR $a b s / 1603.08983$ (2016)

[17] Gruslys, A., Munos, R., Danihelka, I., Lanctot, M., and Graves, A. Memoryefficient backpropagation through time. CoRR abs/1606.03401 (2016)

[18] Hascoët, L., AND PAscual, V. The Tapenade automatic differentiation tool: 
principles, model, and specification. ACM Transactions On Mathematical Software 39,3 (2013).

[19] Hochreiter, S., AND Schmidhuber, J. Long short-term memory. Neural computation 9, 8 (1997), 1735-1780. deeplearning.cs.cmu.edu/pdfs/Hochreiter97 lstm.pdf.

[20] Jia, Y., Shelhamer, E., Donahue, J., Karayev, S., Long, J., Girshick, R., Guadarrama, S., AND Darrell, T. Caffe: Convolutional architecture for fast feature embedding. In Proceedings of ACM Multimedia (2014), pp. 675-678 arxiv.org/abs/1408.5093.

[21] JouppI, N. Google supercharges machine learning tasks with TPU custom chip, 2016. cloudplatform.googleblog.com/2016/05/Google-supercharges-machinelearning-tasks-with-custom-chip.html.

[22] Jouppi, N. P., Young, C., Patil, N., Patterson, D., Agrawal, G., Bajwa, R., Bates, S., Bhatia, S., Boden, N., Borchers, A., Boyle, R., Cantin, P.--., Chao, C., Clark, C., Coriell, J., Daley, M., Dau, M., Dean, J., Gelb, B., Ghaemmaghami, T. V., Gottipati, R., Gulland, W., Hagmann, R., Ho, C. R., Hogberg, D., Hu, J., Hundt, R., Hurt, D., Ibarz, J., Jaffey, A., Jaworski, A., Kaplan, A., Khaitan, H., Killebrew, D., Koch, A., Kumar, N., Lacy, S., Laudon, J., Law, J., Le, D., Leary, C., Liu, Z., Lucke, K., Lundin, A., MacKean, G., Maggiore, A., Mahony, M., Miller, K., Nagarajan, R., Narayanaswami, R., Ni, R., Nix, K., Norrie, T., Omernick, M., Penukonda, N., Phelps, A., Ross, J., Ross, M., Salek, A., Samadiani, E., Severn, C., Sizikov, G., Snelham, M., Souter, J., Steinberg, D., Swing, A., Tan, M., Thorson, G., Tian, B., Toma, H., Tuttle, E., Vasudevan, V., Walter, R., WANG, W., Wilcox, E., AND Yoon, D. H. In-datacenter performance analysis of a Tensor Processing Unit. In Proceedings of the 44th Annual International Symposium on Computer Architecture (New York, NY, USA, 2017), ISCA '17, ACM, pp. $1-12$.

[23] Kudlur, M. Documentation for imperative mode. 2017.

[24] Looks, M., Herreshoff, M., Hutchins, D., AND Norvig, P. Deep learning with dynamic computation graphs. CoRR abs/1702.02181 (2017).

[25] Maclaurin, D., Duvenaud, D., AND Adams, R. P. Autograd: Reverse-mode differentiation of native python. In ICML workshop on Automatic Machine Learning (2015).

[26] Mnih, V., Kavukcuoglu, K., Silver, D., Rusu, A. A., Veness, J., Bellemare, M. G., Graves, A., Riedmiller, M., Fidjeland, A. K., Ostrovski, G., Petersen, S., Beattie, C., Sadik, A., Antonoglou, I., King, H., Kumaran, D., Wierstra, D., LEGG, S., AND Hassabis, D. Human-level control through deep reinforcement learning. Nature 518, 7540 (02 2015), 529-533. dx.doi.org/10.1038/nature14236.

[27] Murray, D. G., McSherry, F., IsAacs, R., Isard, M., Barham, P., AND Abadi, M Naiad: a timely dataflow system. In Proceedings of SOSP (2013), ACM, pp. 439-455. research.microsoft.com:8082/pubs/201100/naiad_sosp2013.pdf.

[28] Murray, D. G., McSherry, F., Isard, M., IsAacs, R., Barham, P., ANd Abadi, M. Incremental, iterative data processing with timely dataflow. Commun. ACM 59, 10 (Sept. 2016), 75-83. dl.acm.org/citation.cfm?id=2983551.

[29] Murray, D. G., Schwarzkopf, M., Smowton, C., Smit, S., Madhavapeddy, A., AND HAND, S. CIEL: A universal execution engine for distributed data-flow computing. In Proceedings of the Ninth USENIX Symposium on Networked Systems Design and Implementation (2011). Usenix PDF.

[30] Nishihara, R., Moritz, P., Wang, S., Tumanov, A., Paul, W., Schleier-Smith, J. Liaw, R., JoRdAN, M. I., AND StoicA, I. Real-time machine learning: The missing pieces. In Proceedings of HotOS (2017). arxiv.org/abs/1703.03924.

[31] PARR, T., AND HowARD, J. The matrix calculus you need for deep learning. arXiv preprint 1802.01528 (2018). arxiv.org/abs/1802.01528.

[32] Pearlmutter, B. A., And Siskind, J. M. Reverse-mode AD in a functional framework: Lambda the ultimate backpropagator. ACM Trans. Program. Lang. Syst. 30, 2 (2008), 7:1-7:36.

[33] Plotkin, G. Some principles of differentiable programming languages, 2018. www.youtube.com/watch?v=qhPBfysSYI8.

[34] PyToRch, 2018. pytorch.org.

[35] Rumelhart, D. E., Hinton, G. E., and Williams, R. J. Learning representations by back-propagating errors. In Cognitive modeling, vol. 5. MIT Press, 1988, pp. 213-220. www.cs.toronto.edu/ hinton/absps/naturebp.pdf.

[36] SEIDE, F., AND AgArwal, A. CNTK: Microsoft's open-source deep-learning toolkit. In $K D D$ (2016), B. Krishnapuram, M. Shah, A. J. Smola, C. Aggarwal, D. Shen, and R. Rastogi, Eds., ACM, p. 2135

[37] Shankar, A., ANd Dobson, W. Eager execution: An imperative, define-by-run interface to TensorFlow, 2017. research.googleblog.com/2017/10/eager-executionimperative-define-by.html.

[38] Shazeer, N., Mirhoseini, A., Maziarz, K., Davis, A., Le, Q. V., Hinton, G. E., AND DEAN, J. Outrageously large neural networks: The sparsely-gated mixtureof-experts layer. CoRR abs/1701.06538 (2017)

[39] SONNET. github.com/deepmind/sonnet.

[40] Sutskever, I., Vinyals, O., ANd Le, Q. V. Sequence to sequence learning with neural networks. In Proceedings of NIPS (2014), pp. 3104-3112. papers.nips.cc/paper/5346-sequence-to-sequence-learning-with-neural.pdf.

[41] SyntaxNet. github.com/tensorflow/models/tree/master/syntaxnet.

[42] TensonFlow. v1.6, www.tensorflow.org/versions/r1.6/api docs.

[43] TensorFlow Fold. github.com/tensorflow/fold.
[44] Tokui, S. Chainer: A powerful, flexible and intuitive framework of neural networks, 2018. chainer.org.

[45] Wu, Y., Schuster, M., Chen, Z., Le, Q. V., Norouzi, M., Macherey, W., Krikun, M., CaO, Y., Gao, Q., Macherey, K., Klingner, J., Shah, A., Johnson, M., Liu, X., Kaiser, L., Gouws, S., Kato, Y., Kudo, T., Kazawa, H., Stevens, K., Kurian, G., Patil, N., Wang, W., Young, C., Smith, J., Riesa, J., Rudnick, A., Vinyals, O., Corrado, G., Hughes, M., And DeAn, J. Google's Neural Machine Translation system: Bridging the gap between human and machine translation. arXiv preprint 1609.08144 (2016). arxiv.org/abs/1609.08144.

[46] XLA. Accelerated linear algebra. www.tensorflow.org/performance/xla. 